\title{
LncRNA-CCAT1/miR-152-5p is Involved in CSE- Induced Inflammation in HBE Cells Via Regulating ERK Signaling Pathway
}

\author{
Dan-dan Zong \\ Second Xiangya Hospital \\ Xiang-ming Liu \\ Second Xiangya Hospital \\ Jin-hua Li \\ Second Xiangya Hospital \\ Ying-jiao Long \\ Second Xiangya Hospital \\ Ruo-yun Ouyang \\ Second Xiangya Hospital \\ Yan Chen ( $\nabla$ chenyan99727@csu.edu.cn ) \\ Second Xiangya Hospital https://orcid.org/0000-0002-7713-6913
}

\section{Research}

Keywords: CCAT1, miR-152-3p, ERK signaling pathway, inflammation, COPD

Posted Date: October 5th, 2021

DOI: https://doi.org/10.21203/rs.3.rs-940217/v1

License: () (1) This work is licensed under a Creative Commons Attribution 4.0 International License. Read Full License 


\section{Abstract}

Background: Emerging studies have noted that dysregulated long non-coding RNAs (IncRNAs) are implicated in the pathological processes of chronic obstructive pulmonary disease (COPD). LncRNA colon cancer-associated transcript 1 (CCAT1) plays well-defined roles in the inflammatory progression. The study aims to figure out the effect and regulatory mechanism of CCAT1 in the cigarette smoke induced inflammation in COPD.

Methods: The expression levels of CCAT1 and miR-152-3p were detected by quantitative real-time polymerase chain reaction (qRT-PCR). The inflammatory levels of IL-1 $\beta$ and IL- 6 were evaluated by qRTPCR and enzyme-linked immunosorbent assay (ELISA). Western blot was used for the measurement of ERK1/2, p-ERK1/2 protein levels. Luciferase reporter assay was performed for the target gene analysis.

Results: CCAT1 was highly expressed in lung tissues of smokers with COPD compared with non-smokers without COPD samples. In human bronchial epithelial (HBE) cells, cigarette smoke extract (CSE) treatment led to an increase in CCAT1 expression in a dose- and time- dependent manner. Functional experiments showed that knockdown of CCAT1 ameliorated CSE-induced inflammation. Mechanistically, CCAT1 directly targeted miR-152-3p, and miR-197-3p overexpression reversed the pro-inflammatory effects of CCAT1 on HBE cells. Subsequently, miR-152-3p was found to regulate ERK signaling pathway. PD98059, ERK specific inhibitor, reversed miR-152-3p inhibition mediated inflammation in HBE cells. In addition, CCAT1 acted as a sponge for miR-152-3p to positively regulate ERK signaling pathway.

Conclusion: Current findings suggest that CCAT1 promoted inflammation by activating ERK signal pathway via sponging miR-152-3p in CSE-treated HBE cells. These results may provide a novel therapeutic target for alleviating cigarette smoke mediated airway inflammation.

\section{Background}

Cigarette smoke is a complex aerosol consisting of more than 4500 identified chemical compounds, which have various toxic, mutagenic and carcinogenic effects [1]. The airway epithelium is the lung's first line of defense and constitutes an essential protection to inhaled insults. Toxic particles of inhaled smoke induce airway inflammation, which plays an essential role in the occurrence and progress of multiple respiratory diseases, such as chronic obstructive pulmonary disease (COPD), and lung cancer [2]. Understanding the impact of airway inflammation induced by cigarette smoke and which mechanism modulates this process may provide new insight into signaling pathways and indicating novel therapeutic targets to control inflammation.

Long non-coding RNA (IncRNA) are a class of non-coding RNA molecules with a length of $>200$ nucleotides that will not be translated into proteins, and they exert their physiological and pathological functions by interacting with genomic DNA, microRNAs (miRNAs), mRNAs, and proteins [3]. LncRNAs have been identified as essential regulators in numerous biological processes, such as cell proliferation, differentiation, apoptosis, and inflammatory response [4]. Several studies have recently shown that 
exposure to cigarette smoke in both humans and rats lead to global alterations in IncRNA expression [5, 6], indicating the potential role of IncRNAs as a novel group of targets for the treatment of cigarette smoke-related lung diseases. Colon cancer-associated transcript 1 (CCAT1), a IncRNA of about $11 \mathrm{~kb}$ located on chromosome 8q24.21, was one of the first IncRNAs that were revealed to play functional roles in the pathogenesis of different types of human cancers, including lung cancer [7]. In cigarette smoke extract (CSE) induced human bronchial epithelial (HBE) cells, CCAT1 expression was significantly increased [8]. Current research has revealed that CCAT1 is associated with inflammatory response in intestinal epithelial cells [9]. Moreover, CCAT1 knockdown significantly alleviated LPS induced expression of pro-inflammatory factors in skin keratinocyte HaCaT cells. It is therefore rational to propose that CCAT1 might exhibit a role in cigarette smoke-induced airway inflammation.

In the present study, we used HBE cells under in vitro CSE conditions to mimic cigarette smoking insult to reveal the effects and molecular mechanisms of CCAT1 in the cigarette smoke induced airway inflammation. We assessed the expression level of CCAT1 in cell lines and examined its effects on the expression of inflammatory genes. Furthermore, we explored the target genes of CCAT1 and the underlying mechanism of its function. This study will provide a better understanding of the pathogenesis of cigarette smoke induced airway inflammation.

\section{Materials And Methods}

\section{Lung tissue Acquirement}

This study was approved by the Institutional Ethics Committee of the Second Xiangya Hospital of Central South University. We recruited consecutive patients from April 2018 to September 2019. Human lung tissue from patients with COPD $(n=10)$ and controls $(n=10)$ was obtained from patients undergoing surgery and who gave informed consent. All the 20 patients were enrolled with primary lung cancer of stage 1 and had the surgery of pulmonary segmentectomy or lobectomy. All the patients were clinically stable for 4 weeks without acute pulmonary infection, did not receive chemotherapy half a year before the study, and did not have obstructive atelectasis, metastasis, other pulmonary diseases, and severe diseases in other systems. The lung tissue $5 \mathrm{~cm}$ away from tumor margin was used in our studies. The pathological examination confirmed that these samples presented lung structure without metastasis or inflammation.

\section{Cell lines and cell culture}

HBE cells were cultured in RPMI 1640 (Gibco, C11875500BT) supplemented with 10\% fetal bovine serum (Gibco, 10082147), 100U/ml penicillin and $100 \mu \mathrm{g} / \mathrm{ml}$ streptomycin (Thermo Fisher Scientific, Waltham, MA, USA) in $5 \% \mathrm{CO} 2$ atmosphere at $37^{\circ} \mathrm{C}$. Cells were passaged at $80 \%$ confluence and grown to full confluence for the experiments. All experiments were performed in triplicate and repeated independently at least three times. After serum starvation for $24 \mathrm{~h}$, the HBE cells were treated with CSE at the indicated concentrations. 


\section{Preparation of CSE}

CSE was prepared as previously described [10]. Briefy, one nonfiltered Fu-Rong cigarette (Furong, Changde Cigarette Company, Hunan, China) was burned, and the smoke was passed through $20 \mathrm{ml}$ of phosphate-bufered saline via a vacuum pump. This $100 \%$ CSE solution was adjusted to $7.2-7.4$ and filtered through a $0.22 \mathrm{~m}$ membrane filter to remove large particles and bacteria before use. Then, CSE was diluted with PBS to obtain concentrations of $1 \%, 2.5 \%$, and $5 \%$. CSE was freshly prepared within the 30 min preceding each experiment.

\section{Quantitative Real-Time polymerase chain reaction (qRT- PCR)}

Total RNA was isolated and extracted from different groups of lung tissues and cells by adding TRIzol (Life Technologies, Carlsbad, CA), chloroform, isopropanol, and 75\% ethanol. Single-stranded cDNA was synthesized from $1 \mu \mathrm{g}$ RNA using a reverse reaction kit (Thermo Fisher Scientific. Waltham, MA, USA). Then qRT-PCR was performed on an ABI 7500 instrument (Applied Biosystems, Foster City, CA) using iTapTM SYBR Green Supermix with ROX dye (Bio-Rad Laboratories, Hercules, CA). The primers for miR152-3p and U6 were obtained from RiboBio Co. Ltd. (Guangdong, China). The forward and reverse primers for other genes (RiboBio, Guangzhou, China) are listed as follows:

CCAT1, forward 5'-CACCTACGCATACCTCTGCTTC-3', reverse 5'-TGATTGCTCCTGTTTCCCTTTG-3'; IL-1 $\beta$, forward 5'-GAAACCCTCTGTCATTCGCTC-3', reverse 5'-CAGACACTGCTACTTCTTGCCC-3'; IL-6, forward 5'TGCCAGCCTGCTGACGAA-3', reverse 5'-AGCTGCGCAGAATGAGATGA-3'; GAPDH, forward 5'GAACGGGAAGCTCACTGG-3', reverse 5'- GCCTGCTTCACCACCTTCT-3'.

\section{Cell Transfection}

miR-152-3p mimic, miR-152-3p inhibitor, mimic negative control (mimic-NC), inhibitor negative control (inhibitor-NC), small interfering RNAs (siRNAs) against CCAT1 (si-CCAT1) and negative control siRNA (siNC) were purchased from RiboBio (RiboBio, Guangzhou, China). CCAT1-overexpression vector (CCAT1) and empty vector (CCAT1-NC) were available from GeneChem (Shanghai, China). They were transfected into HBE cells by Lipofectamine 3000 (Thermo Fisher Scientific. Waltham, MA, USA), according to the manufacturer's protocols.

\section{Western blot analysis}

Total proteins from cells were extracted using RIPA lysis buffer (Beyotime, Shanghai, China). Protein concentrations of all groups were determined by a BCA protein assay kit (Thermo Fisher Scientific, Waltham, MA, USA). Proteins were separated by $10 \%$ SDS-PAGE, then transferred to PVDF membranes and incubated with specific primary antibodies against t-ERK (ab184699, Abcam, Cambridge, UK), p-ERK (ab201015, Abcam, Cambridge, UK), and $\beta$-actin (10068-1-AP, Proteintech, Wuhan, China) overnight at $4^{\circ} \mathrm{C}$. The membrane was incubated with HRP-labelled secondary antibody (Proteintech, Wuhan, China) for 
$1 \mathrm{~h}$ at room temperature. An enhanced chemiluminescence detection system (BIO-RAD, California, USA) was used to detect the protein bands.

\section{Enzyme-Linked Immunosorbent Assay (ELISA)}

Following appropriate CSE treatment and indicated transfection, the concentrations of interleukin-1 $\beta$ (IL$1 \beta$ ) and IL- 6 from the culture supernatants of HBE cells were assayed using commercial the ELISA kits (Proteintech, Wuhan, China) referring to the manufacturer's protocols. The results represent as the average of three independent replicates.

\section{Dual-Luciferase Reporter Assay}

The CCAT1 fragment with the predicted binding site of miR-152-3p was synthesized and cloned into the luciferase reporter gene to form the reporter vector CCAT1-wild-type (CCAT1-Wt). CCAT1-miR-152-3p binding site was mutated as instructed and named CCAT1 mutant (CCAT1-Mut). MiR-152-3p mimic and mimic-NC were co-transfected with CCAT1-Wt or CCAT1-Mut into HEK293T cells, respectively. After 48 h, the relative luciferase activity of each well was measured using the Dual Luciferase Reporter System (Promega, Madison, WI, United States).

\section{Statistical analysis}

Quantitative data were presented as mean \pm SD taken from at least three independent experiments. For relative gene expression, the mean value of the vehicle control group was defined as 1 or $100 \%$. SPSS 21.0 (SPSS, Chicago, IL, USA) software was used for statistical analysis. Student's $t$-test and one-way analysis of variance were used for the comparison of the statistical differences between groups. Tukey's test or Dunnett's T3 test was used for post hoc multiple comparisons according to the homogeneity test of variance. Significance was defined as $p<0.05$.

\section{Results}

\section{CCAT1 expression was upregulated in lung tissues of COPD patients and CSE-induced HBE cells}

To reveal the role of CCAT1 in COPD, we detected their expression levels in COPD patients and HBE cells. Results showed that CCAT 1 expression was significantly upregulated in smokers with COPD as compared with non-smokers without COPD (Fig. 1A). Then, we detected the expression of CCAT1 in CSE treated HBE cells. After exposing HBE cells to CSE concentrations of $0.0 \%, 1.0 \%, 2.5 \%$, and $5.0 \%$ for $24 \mathrm{~h}$, the expression CCAT1 was increased in a dose- dependent manner (Fig. 1B). Finally, we incubated HBE cells with $5 \%$ CSE, a time- related increase of CCAT1 expression was observed from $6 \mathrm{~h}$ (Fig. 1C). These data suggest a potential role for CCAT1 in COPD pathogenesis.

\section{CCAT1 mediates CSE induced inflammation}


First, HBE cells were treated with different concentrations of CSE for different time. Gradually elevated expression and secretion of inflammatory factor were observed (Fig. 2A-2H). Then, we examined the effect of CCAT1 on inflammation with a loss-of-function approach. The cells were transfected with siCCAT1. The results shown that CCAT1 expression was increased after CSE treatment, whereas this effect was attenuated by si-CCAT1 transfection (Fig. 2I). Inflammatory mediators IL-1 $\beta$ and IL- 6 levels were upregulated in CSE-induced HBE cells, and these influences were alleviated by si-CCAT1 (Fig. 2J-2M).

\section{CCAT1 modulates miR-152-3p expression}

We predicted the potential target miRNA of CCAT1 by online software StarBase, and found that there were binding sites of miR-152b-3p with CCAT1 (Fig. 3A). Luciferase reporter assay demonstrated that overexpression of miR-152b-3p significantly repressed the luciferase activity of CCAT1-Wt but not CCAT1Mut (Fig. 3B). Next, miR-152-3p expression was further measured using qRT-PCR, which suggested that miR-152-3p expression was reduced in the lung tissues of smokers with COPD compared to non-smokers without COPD (Fig. 3C). In addition, CSE treatment led to a decrease in miR-152-3p expression in HBE cells in a dose- and time- dependent manner (Fig. 3D,3E). We further investigated the effects of CCAT1 upregulation on miR-152-3p expression in HBE cells. The results showed that CCAT1 upregulation reduced miR-152-3p expression (Fig. 3F,3G). These results confirmed that CCAT1 targeted miR-152-3p and negatively regulated its expression.

\section{The proinflammatory effect of CCAT1 in HBE cells was dependent on miR-152-3p}

First, miR-152-3p mimics were transfected into HBE cells to investigate its role in inflammation. qRT-PCR analysis showed that miR-152-3p mimic significantly rescued the decrease of miR-152-3p expression induced by CSE (Fig. 4A). As the miR-152-3p level restored, the expression and secretion of inflammatory factors IL-1 $\beta$ and IL- 6 decreased markedly (Fig. 4B-4E). Then, we explored whether miR-152-3p mediated the proinflammatory effect of CCAT1 on CSE treated HBE cells. CCAT1 overexpression vector and miR152-3p mimic were co-transfected into HBE cells. As shown in the result, when CCAT1 vector was transfected, the level of CCAT1 increased significantly compared to CCAT1-NC group (Fig. 4F), meanwhile the inflammatory level increased. MiR-152-3p mimic rescued the decreased level of miR-152-3p induced by CCAT1 overexpression (Fig. 4G), and led to a corresponding reduction of inflammation (Fig. 4H-4K). These results suggest that CCAT1 regulate inflammation at least partly via targeting miR-152-3p and restoration of miR-152-3p can relieve the pro-inflammatory effect of CCAT1 in HBE cells.

\section{CSE triggers inflammation via extracellular signal-regulated kinase (ERK) signaling pathway}

Our previous study has confirmed that ERK signaling pathway was activated in lung tissue of COPD patients [11]. The role of ERK signaling on CSE-induced inflammation in HBE cells was then elucidated. 
As expected, the expression level of p-ERK1/2 was substantially increased in HBE cells exposed to CSE, while this upregulation was inhibited by specific ERK1/2 inhibitor, PD98059 (Fig. 5A,5B). After that, the inflammatory level was detected. The results showed that PD98059 significantly attenuated CSEtriggered elevation of IL-1 $\beta$ and IL-6 in 16HBE cell (Fig. 5C-5E).

\section{miR-152-3p regulates inflammation in HBE cells by modulating ERK signaling pathway}

Whether miR-152-3p was responsible for inflammatory response in HBE cells via ERK signaling pathway was then investigated. We decreased the expression of miR-152-3p in HBE cells by transfecting with miR152-3p inhibitor (Fig. 6A). Western blot analysis revealed the decrease in miR-152-3p promoted upregulated p-ERK1/2 protein (Fig. 6B.6C), and led to a corresponding induction of inflammatory levels (Fig. 6D-6G). In contrast, treatment of HBE cells with PD98059 suppressed the IL-1 $\beta$ and IL-6 expression and secretion induced by transfection with miR-152-3p inhibitor (Fig. 6D-6G).

\section{CCAT1 activates ERK signaling pathway via targeting miR- 152-3p}

Finally, we detected whether CCAT1 could regulate ERK signaling pathway via targeting miR-152-3p. As shown in Fig. 7A and Fig. 7B, ERK signaling pathway was activated by CCAT1 overexpression, while these impacts were attenuated by miR-152-3p mimic in HBE cells, indicating that CCAT1 modulated ERK signaling pathway in an miR-152-3p- dependent manner. Altogether, this study demonstrated that CCAT1 activate ERK signaling pathway via targeting miR-152-3p to promote CSE-induced inflammation, thus involving in COPD process.

\section{Discussion}

Cigarette smoking is a major preventable risk factor for COPD. Chronic airway inflammation induced by cigarette smoke exposure was previously reported to be one of the key pathogenic mechanisms. However, the mechanism remains largely unknown, which is also the reason why this experiment is designed. In the current study, we observed that IncRNA CCAT1 was highly expressed in smokes with COPD and in CSE treated HBE cells. Silencing CCAT1 alleviated inflammation in HBE cells, suggesting a potential therapeutic target of CCAT1 for CS-induced airway inflammation.

Emerging evidence has suggested that IncRNAs are abnormal expressed in several pulmonary disorders, implying the potential role of IncRNAs in the pathogenesis of these pulmonary diseases [12, 13]. Recently, several studies had revealed that IncRNAs were identified as a pivotal modulator in the inflammatory process $[14,15]$. Therefore, research on IncRNA may help improve the diagnosis and treatment of lung inflammatory diseases, such as COPD. Furthermore, previous studies revealed that IncRNAs differentially expressed in lung tissue from non-smokers and smokers without or with COPD $[5,16]$. As a relatively wellinvestigated IncRNA, CCAT1 has been demonstrated to facilitate cell proliferation and inhibit apoptosis in 
lung cancer [17]. In addition, CCAT1 expression was upregulated in CSE treated HBE cells. Nonetheless, no further study on the function of CCAT1 in COPD have been studied. In the previous study, we showed that the expression of CCAT1 was obviously increased in COPD patients. Subsequently, we identified that CSE promoted CCAT1 expression in a dose- and time- dependent manner in HBE cells. Besides, we explored the effects of CCAT1 on inflammatory response. Results showed that knockdown of CCAT1 attenuated CSE-induced pro-inflammatory effects on HBE cells in vitro. These data suggested that CCAT1 was involved in COPD development and progression.

MiRNAs are a family of small non-coding RNAs with the length of 18-22 nucleotides. They can regulate the expression of one or more genes after transcription and participate in various physiological and pathological processes, which are closely related to the COPD [18]. Accumulating evidence has indicated that IncRNAs can play the role of endogenous miRNA sponge, and then inhibit miRNA expression, thereby modulating a variety of cellular biological activities [19]. In this study, we predicted that there were regulatory sites between miR-152-3p and CCAT1 through online software StarBase. Then we conducted a series of experiments to further confirm that CCAT1 could directly bind to miR-152-3p and negatively modulated its expression in HBE cells. Previous study has shown that miR-152 overexpression attenuated doxorubicin-induced inflammation, whereas miR-152 knockdown resulted in inflammation [20], suggesting a pro-inflammatory effect of miR-152. However, the role of miR-152 in COPD has never been investigated. Here, we found that the miR-152-3p abundance was decreased in the lung tissues of smoker with COPD. In CSE treated HBE cells, we also observed a decrease in miR-152-3p expression accompanied with increased inflammatory factor levels, while upregulation of miR-152-3p reduced CSEinduced inflammatory cytokines release. Moreover, miR-152-3p overexpression reversed the influence of CCAT1 on inflammatory response in CSE-exposed HBE cells. These results indicate that CCAT1 might promote COPD development by sponging miR-152-3p.

ERK is a subclass of the mitogen-activated protein kinase family that plays critical roles in cellular signal transduction. The activated ERK was translocated to the nucleus and phosphorylates multiple substrates to regulate the activity of transcription factors and produce cellular effect [21]. It has been reported that ERK signaling pathway functions as a key mediator of various physiological processes, including inflammatory responses [22]. Besides, previous study has confirmed that miR-152 could inhibit cell proliferation, survival and migration via deactivating ERK signaling pathway [23]. Thus, we hypothesize that miR-152 may regulate CSE induced inflammation via modulating ERK signaling pathway. In the present study, we verified that ERK signaling pathway was activated after CSE exposure, and the inhibition of ERK signaling pathway reduced CSE-stimulated inflammation in HBE cells. Moreover, it was proved that overexpression of miR-152-3p exerted its anti-inflammatory effect on HBE cells by inhibiting ERK signaling pathway. Additionally, it is also demonstrated that CCAT1 could regulate ERK signaling pathway by sponging miR-152-3p in HBE cells.

Taken together, our study suggested that CCAT1 was overexpressed in lung tissues of smokers with COPD and in CSE treated HBE cells. CCAT1 knockdown alleviated CSE-induced inflammation in HBE cells 
by down-regulating ERK signaling pathway through sponging miR-197-3p. These results showed that CCAT1 acts as a potential therapeutic target for further treatment of COPD.

\section{Abbreviations}

CCAT1: colon cancer-associated transcript 1; COPD: chronic obstructive pulmonary disease; CSE: cigarette smoke extract; ERK: extracellular signal-regulated kinase; HBE: human bronchial epithelial; IL-1ß: interleukin; IncRNA: long non-coding RNA; miRNA: microRNA; NC: negative control

\section{Declarations}

\section{Ethics approval and consent to participate}

The studies involving human participants were reviewed and approved by the institutional ethics committee of The Second Xiangya Hospital (2019-006). Written informed consent to participate in this study was provided by the participants' legal guardian/next of kin.

\section{Consent for publication}

Not applicable

\section{Availability of data and materials}

All data generated or analysed during this study are included in this published article.

\section{Competing interests}

The authors declare that they have no competing interests.

\section{Funding}

This study was supported by the National Natural Science Foundation of China (Grant No. 81873410 , 81900042 and 82070049) and the National Key Clinical Specialty Discipline Construction Program of China (Grant No. 2012-650).

\section{Authors' contributions}

Every author contributed to the reviewing of the paper. ZDD and LXM performed the laboratory work, statistical analyses, and drafted the manuscript. LJH and LYJ performed part of the laboratory work and statistical analyses. OYRY supervised the study and helped to revise the manuscript. CY directed and corrected this research as corresponding author. All authors read and approved the final manuscript.

\section{Acknowledgements}

Not applicable

\section{References}


1. Lugg ST, Scott A, Parekh D, Naidu B, Thickett DR. Cigarette smoke exposure and alveolar macrophages: mechanisms for lung disease. Thorax. 2021.

2. Zong D, Liu X, Li J, Ouyang R, Chen P. The role of cigarette smoke-induced epigenetic alterations in inflammation. Epigenetics Chromatin. 2019;12:65.

3. Wang W, Min L, Qiu X, Wu X, Liu C, Ma J, et al. Biological Function of Long Non-coding RNA (LncRNA). Xist Front Cell Dev Biol. 2021;9:645647.

4. Chen Y, Li Z, Chen X, Zhang S. Long non-coding RNAs: From disease code to drug role. Acta Pharm Sin B. 2021;11:340-54.

5. Zhou AY, Zhao YY, Zhou ZJ, Duan JX, Zhu YZ, Cai S, et al. Microarray Analysis of Long Non-Coding RNAs in Lung Tissues of Patients with COPD and HOXA-AS2 Promotes HPMECs Proliferation via Notch1. Int J Chron Obstruct Pulmon Dis. 2020;15:2449-60.

6. Zhang H, Sun D, Li D, Zheng Z, Xu J, Liang X, et al. Long non-coding RNA expression patterns in lung tissues of chronic cigarette smoke induced COPD mouse model. Sci Rep. 2018;8:7609.

7. Wang W, Hou Z, Wen C, Ge L, Ge L. Long Non-coding RNA Colon Cancer-Associated Transcript-1 Promotes Migration, Invasion, and Epithelial Mesenchymal Transition of Lung Adenocarcinoma by Suppressing miR-219-1. Front Genet. 2020;11:929.

8. Lu L, Xu H, Luo F, Liu X, Lu X, Yang Q, et al. Epigenetic silencing of miR-218 by the IncRNA CCAT1, acting via BMI1, promotes an altered cell cycle transition in the malignant transformation of $\mathrm{HBE}$ cells induced by cigarette smoke extract. Toxicol Appl Pharmacol. 2016;304:30-41.

9. Ma D, Cao Y, Wang Z, He J, Chen H, Xiong H, et al. CCAT1 IncRNA Promotes Inflammatory Bowel Disease Malignancy by Destroying Intestinal Barrier via Downregulating miR-185-3p. Inflamm Bowel Dis. 2019;25:862-74.

10. Zong DD, Liu XM, Li JH, Ouyang RY, Long YJ, Chen P, et al. Resveratrol attenuates cigarette smoke induced endothelial apoptosis by activating Notch1 signaling mediated autophagy. Respir Res. 2021;22:22.

11. Zong D, Li J, Cai S, He S, Liu Q, Jiang J, et al. Notch1 regulates endothelial apoptosis via the ERK pathway in chronic obstructive pulmonary disease. Am J Physiol Cell Physiol. 2018;315:C330-40.

12. Poulet C, Njock MS, Moermans C, Louis E, Louis R, Malaise M, et al. Exosomal Long Non-Coding RNAs in Lung Diseases. Int J Mol Sci. 2020; 21.

13. Li Y, Yin Z, Fan J, Zhang S, Yang W. The roles of exosomal miRNAs and IncRNAs in lung diseases. Signal Transduct Target Ther. 2019;4:47.

14. Rikhtegar R, Mosimann PJ, Rothaupt J, Mirza-Aghazadeh-Attari M, Hallaj S, Yousefi M, et al. Noncoding RNAs role in intracranial aneurysm: General principles with focus on inflammation. Life Sci. 2021;278:119617.

15. Chen C, He Y, Feng Y, Hong W, Luo G, Ye Z. Long non-coding RNA review and implications in acute lung inflammation. Life Sci. 2021;269:119044. 
16. Bi H, Zhou J, Wu D, Gao W, Li L, Yu L, et al. Microarray analysis of long non-coding RNAs in COPD lung tissue. Inflamm Res. 2015;64:119-26.

17. Jin X, Liu X, Zhang Z, Guan Y. IncRNA CCAT1 Acts as a MicroRNA-218 Sponge to Increase Gefitinib Resistance in NSCLC by Targeting HOXA1. Mol Ther Nucleic Acids. 2020;19:1266-75.

18. Canas JA, Rodrigo-Munoz JM, Sastre B, Gil-Martinez M, Redondo N. Del Pozo V. MicroRNAs as Potential Regulators of Immune Response Networks in Asthma and Chronic Obstructive Pulmonary Disease. Front Immunol. 2020;11:608666.

19. Huang SF, Peng XF, Jiang L, Hu CY, Ye WC. LncRNAs as Therapeutic Targets and Potential Biomarkers for Lipid-Related Diseases. Front Pharmacol. 2021;12:729745.

20. Zhang WB, Lai X, Guo XF. Activation of Nrf2 by miR-152 Inhibits Doxorubicin-Induced Cardiotoxicity via Attenuation of Oxidative Stress, Inflammation, and Apoptosis. Oxid Med Cell Longev. 2021; 2021:8860883.

21. Gallo S, Vitacolonna A, Bonzano A, Comoglio P, Crepaldi T. ERK: A Key Player in the Pathophysiology of Cardiac Hypertrophy. Int J Mol Sci. 2019; 20.

22. Liang Y, Du R, Chen R, Chu PH, Ip MSM, Zhang KYB, et al. Therapeutic potential and mechanism of Dendrobium officinale polysaccharides on cigarette smoke-induced airway inflammation in rat. Biomed Pharmacother. 2021;143:112101.

23. Ghazanchaei A, Mansoori B, Mohammadi A, Biglari A, Baradaran B. Restoration of miR-152 expression suppresses cell proliferation, survival, and migration through inhibition of AKT-ERK pathway in colorectal cancer. J Cell Physiol. 2018;234:769-76.

\section{Figures}

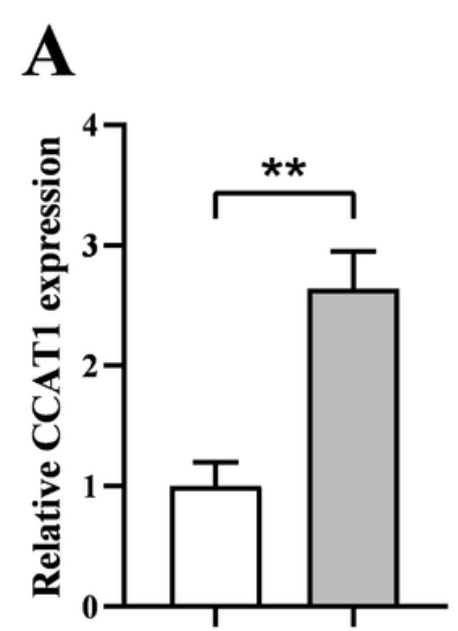

Control COPD
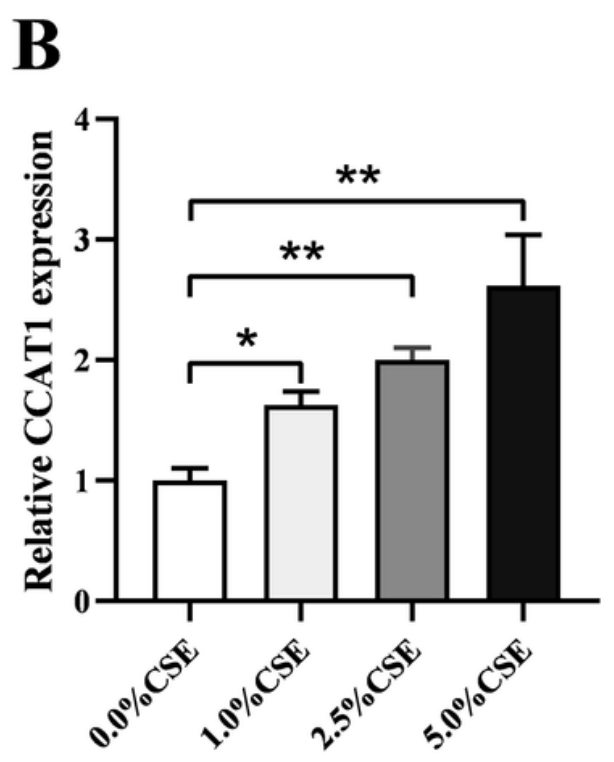
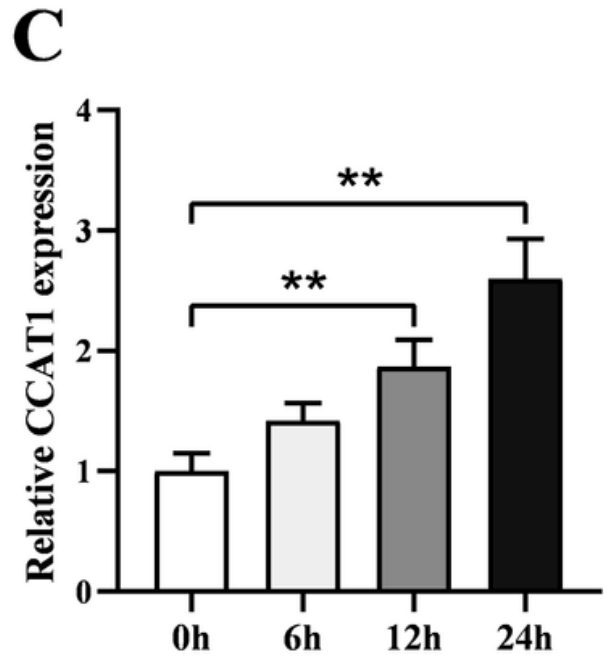
CCAT1 is highly expressed in smokers with COPD and in CSE-induced HBE cells. (A) qRT-PCR assay was used to detect the expression level of CCAT1 in lung tissues of non-smokers without COPD (control group) and smoker with COPD (COPD group). (B, C) The expression level of CCAT1 was measured by qRTPCR assay in HBE cells treated with CSE at different concentrations for $24 \mathrm{~h}$ or exposed to $5 \% \mathrm{CSE}$ for indicated times. Data shown are mean $\pm S D$ and from three independent experiments. ${ }^{*} P<0.05,{ }^{*} P<0.01$.
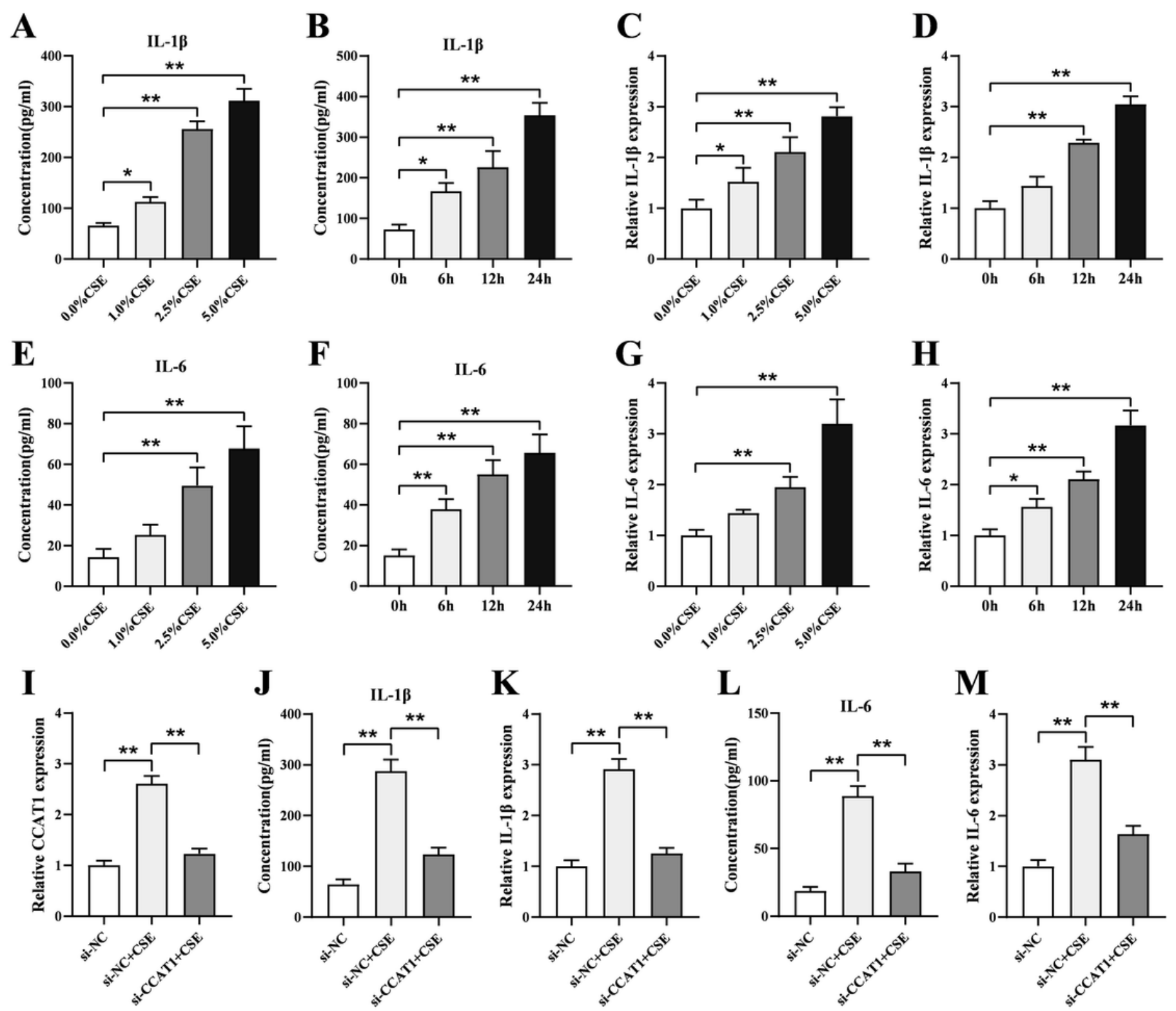

Figure 2

CCAT1 silence alleviated CSE-induced inflammation in HBE cells. (A-H) The levels of inflammatory cytokines IL-1 $\beta$, IL- 6 were detected by ELISA assay and qRT-PCR assay in HBE cells treated with CSE at different concentrations for $24 \mathrm{~h}$ or exposed to $5 \%$ CSE for indicated times. (I) The effects of CSE treatment and si-CCAT 1 transfection on CCAT1 expression were demonstrated by qRT-PCR. (J-M) CCAT1 silence reversed the effects of CSE on expression and release of IL-1 $\beta$ and IL- 6 in HBE cells. Data shown are mean $\pm S D$ and from three independent experiments. ${ }^{*} P<0.05,{ }^{*} \mathrm{P}<0.01$. 


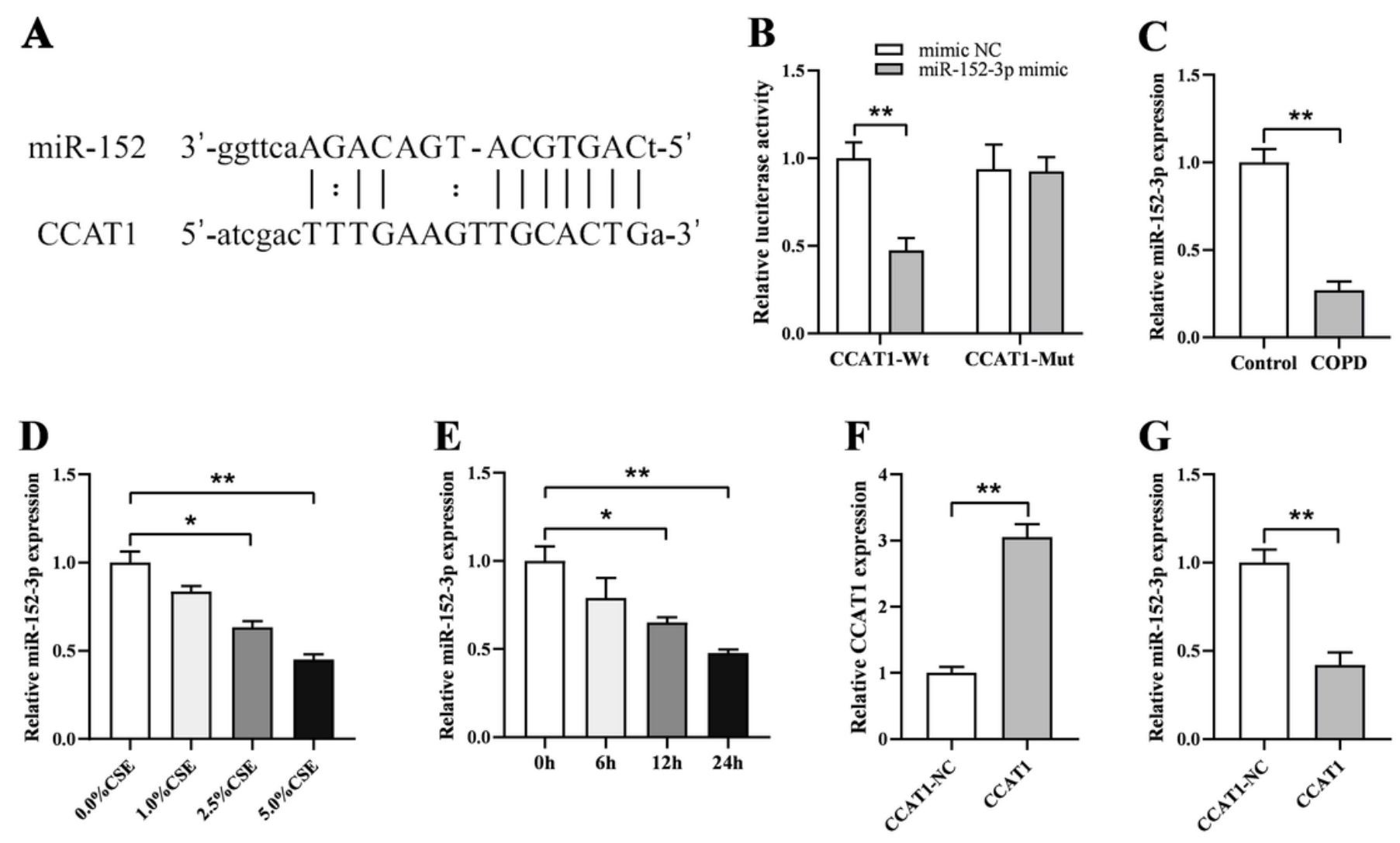

\section{Figure 3}

miR-152-3p was a target of CCAT1 and was downregulated by cigarette smoke. (A) The potential binding sites of miR-152-3p in the CCAT1 were predicted by bioinformatics analysis. (B) Dual-luciferase reporter assay was performed to analyze the relative luciferase activity in HBE cells co-transfected with miR-152$3 p$ mimic or mimic-NC and CCAT1-Wt or CCAT1-Mut. (C) qRT-PCR assay was used to detect the expression level of miR-152-3p in lung tissues of control group and COPD group. (D) The expression level of miR-152-3p was measured by qRT-PCR assay in HBE cells treated with CSE at different concentrations for $24 \mathrm{~h}$ or exposed to $5 \%$ CSE for indicated times. $(F, G)$ The expression level of CCAT1 or miR-152-3p in HBE cells transfected with CCAT1 vector or CCAT1-NC were determined using qRT-PCR assay. Data shown are mean $\pm S D$ and from three independent experiments. ${ }^{*} P<0.05,{ }^{*} P<0.01$. 
A

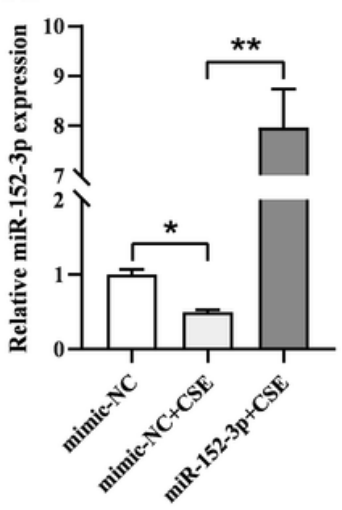

E

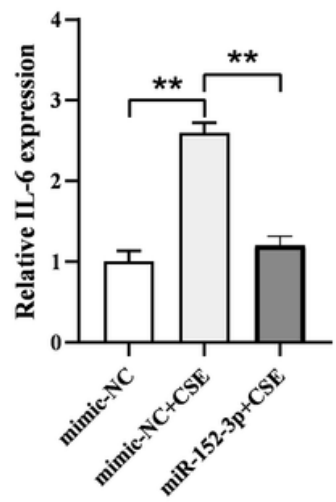

I
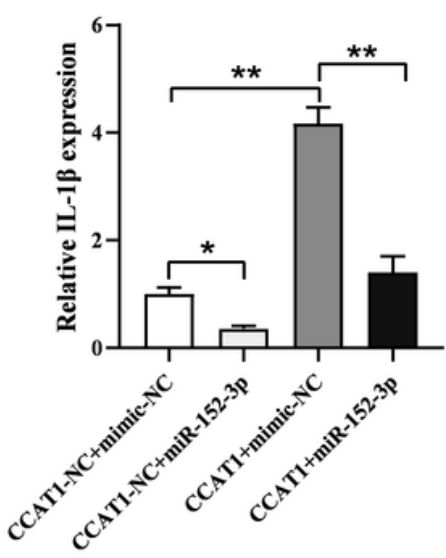

B

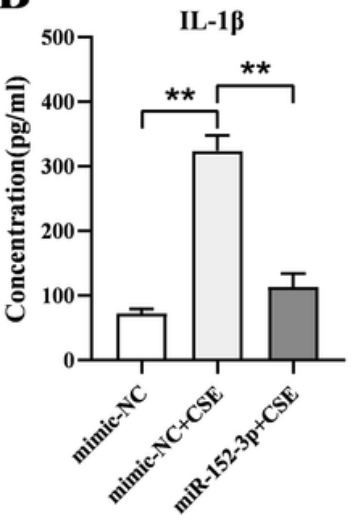

F
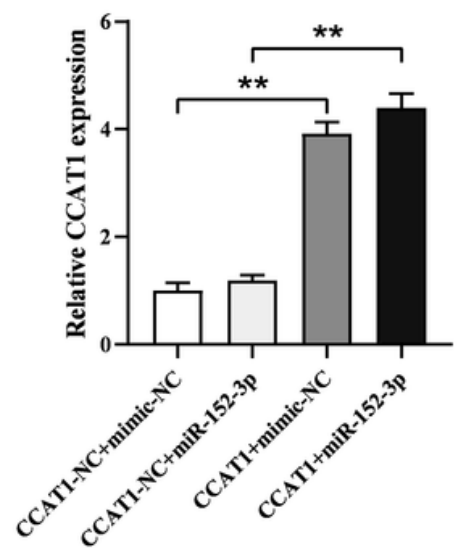

J

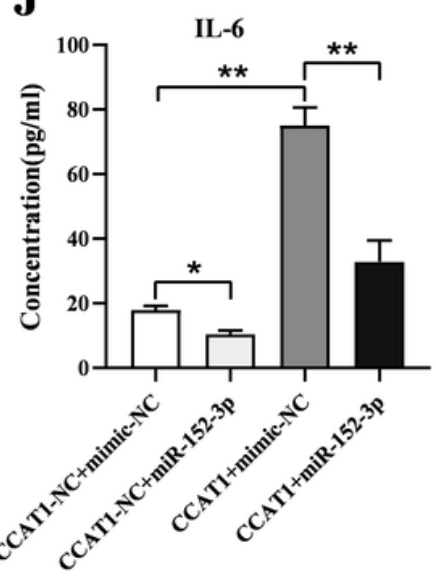

C

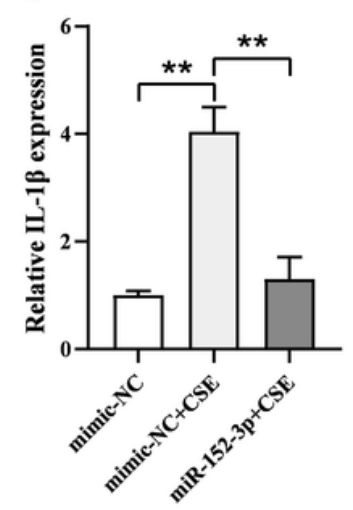

G

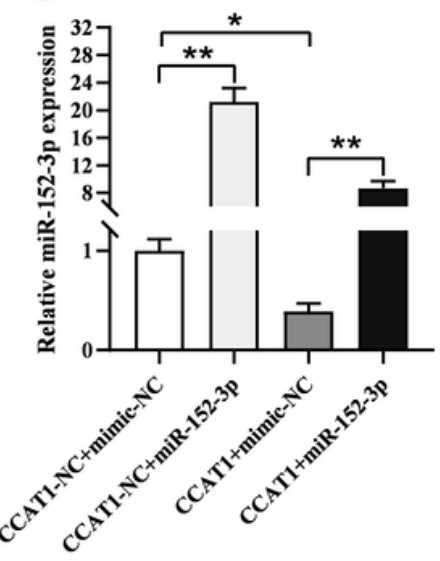

K

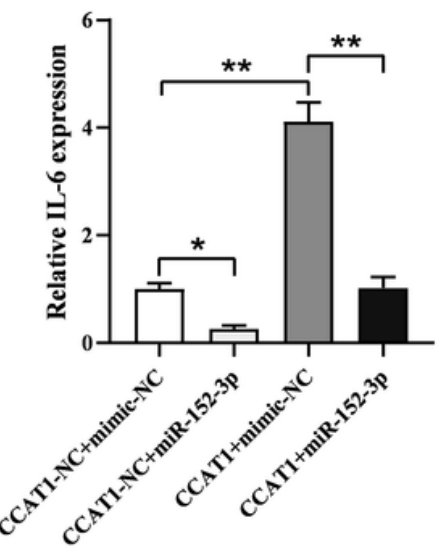

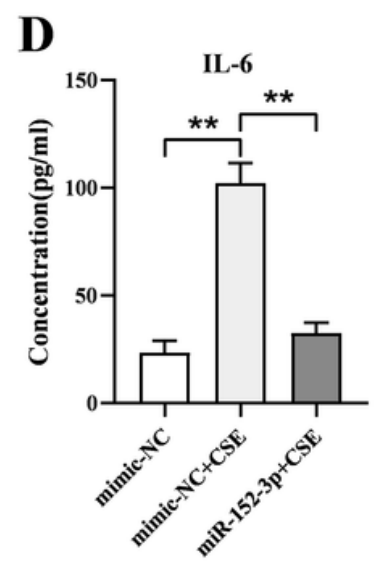

H

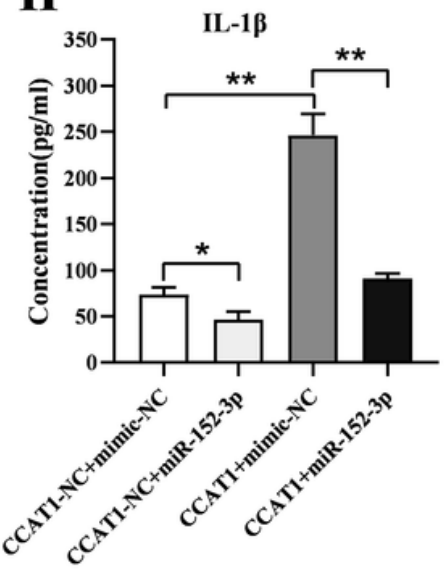

\section{Figure 4}

miR-152-3p overexpression abolished the effect of CCAT1 on inflammation in HBE cells. (A) The effects of CSE treatment and miR-152-3p mimic transfection on miR-152-3p expression were demonstrated by qRT-PCR. (B-E) miR-152-3p overexpression restored the effects of CSE on expression and release of IL-1 $\beta$ and IL-6 in HBE cells. $(F, G)$ qRT-PCR assay was used to measure the expression level of CCAT1 or miR152-3p in HBE cells co-transfected with CCAT1 vector or CCAT1-NC and miR-152-3p mimic or mimic-NC. 
(H-K) miR-152-3p overexpression relieved the pro-inflammatory effect of CCAT1 on HBE cells. Data shown are mean $\pm S D$ and from three independent experiments. ${ }^{*} P<0.05,{ }^{*} \mathrm{P}<0.01$.

$\mathbf{A}$

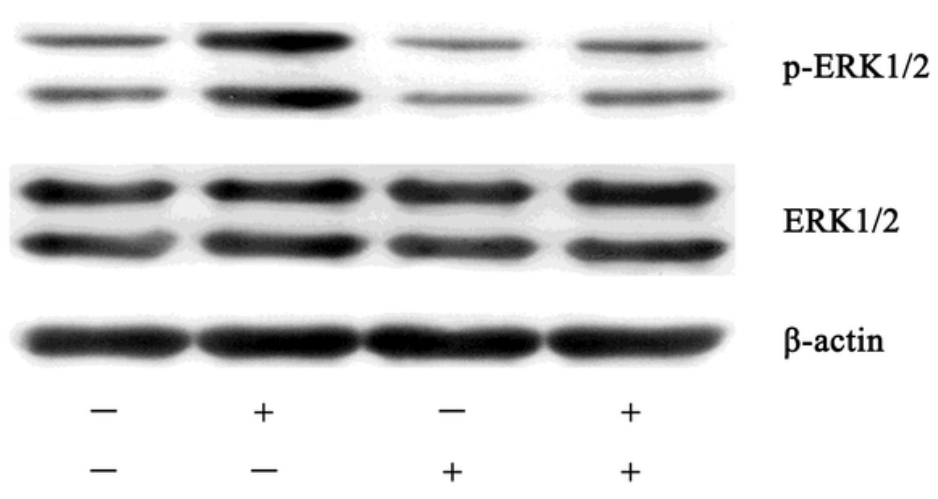

C

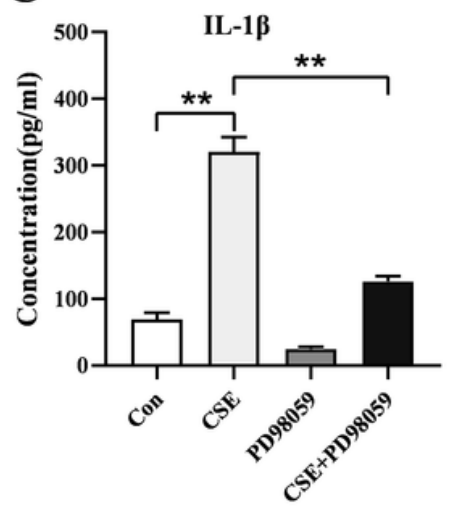

D $\quad$ E

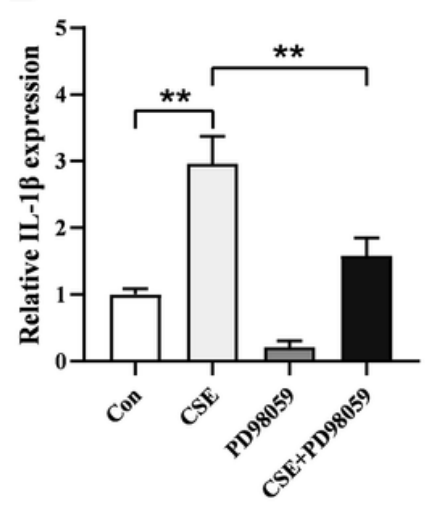

B

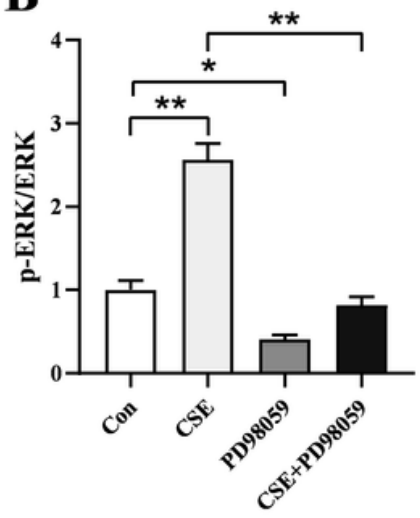

F
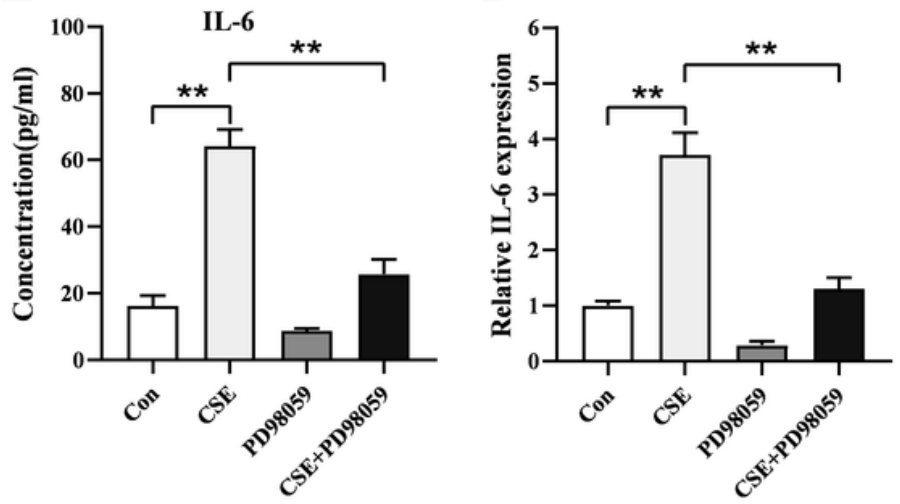

Figure 5

ERK signaling pathway was involved in CSE induced inflammation. (A,B) Protein expression levels of ERK1/2, p-ERK1/2 in each experimental group were analyzed by Western blot. (C-F) PD98059 reversed the effects of CSE on expression and release of IL- $1 \beta$ and IL- 6 in HBE cells. Data shown are mean \pm SD and from three independent experiments. ${ }^{*} P<0.05,{ }^{*} \mathrm{P}<0.01$. 
A

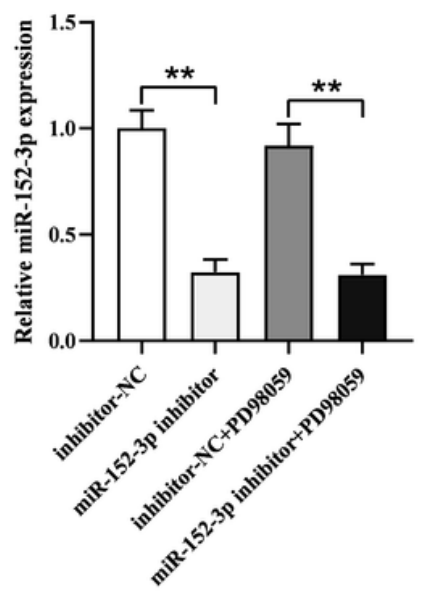

D

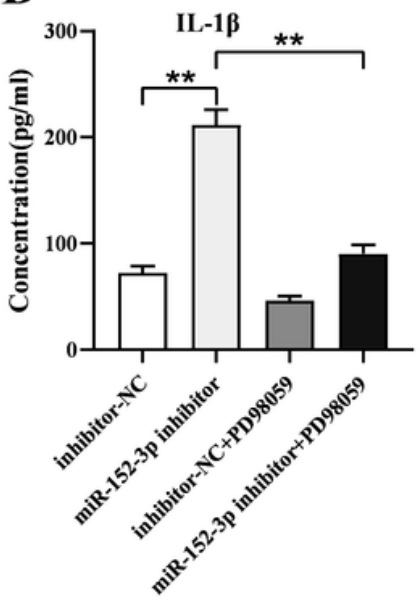

B

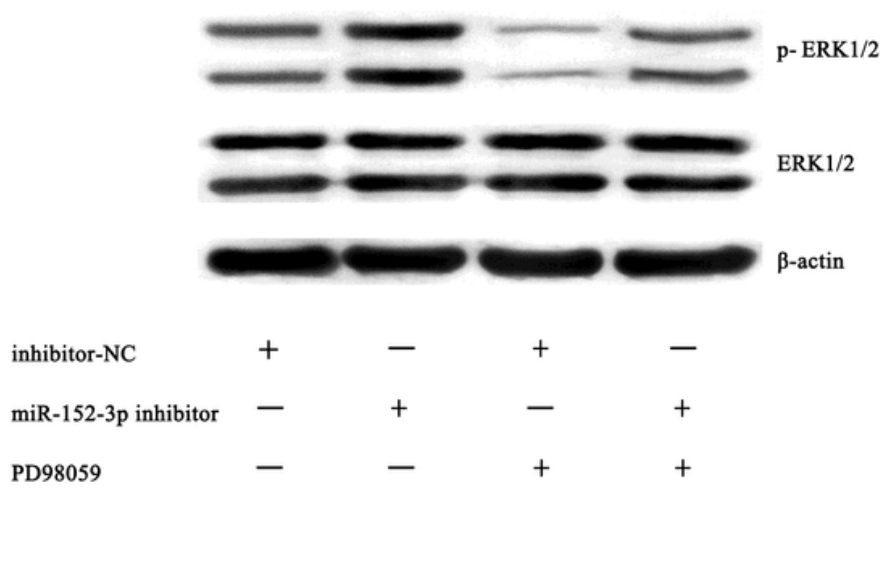

$\mathbf{E}$

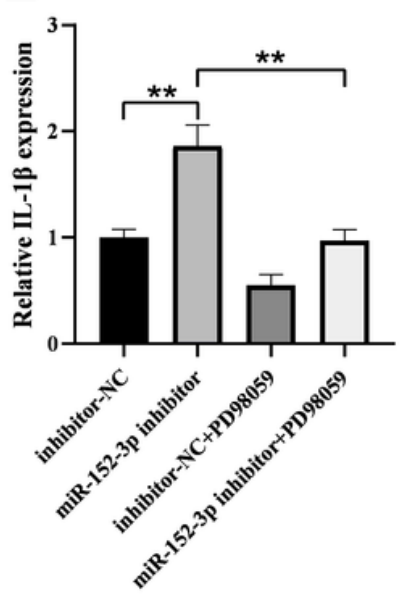

C

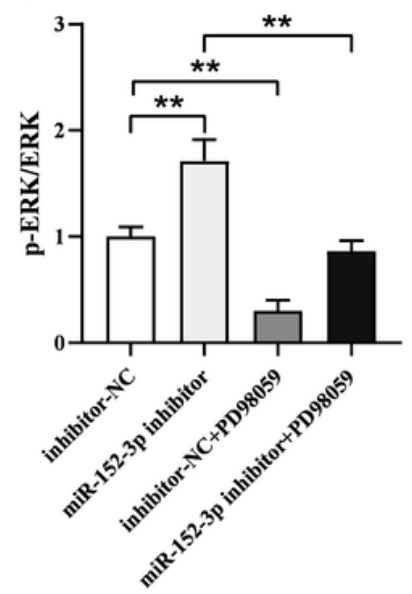

$\mathbf{F}$

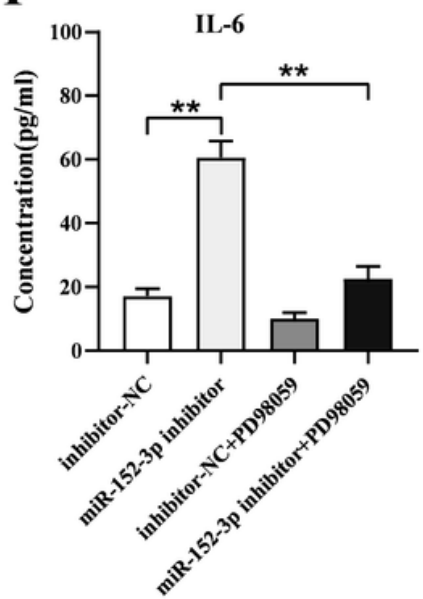

G

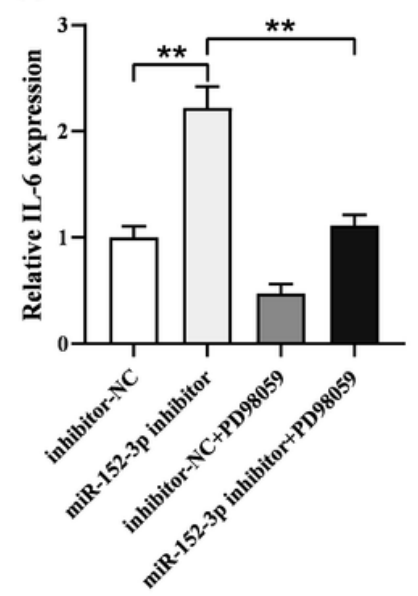

Figure 6

miR-152-3p ameliorated inflammation in HBE cells via regulating ERK signaling pathway. (A) The effects of miR-152-3p inhibitor transfection on miR-152-3p expression were demonstrated by qRT-PCR. (B,C) Protein expression levels of ERK1/2, p-ERK1/2 in each experimental group were analyzed by Western blot. (D-G) PD98059 reduced the inflammatory levels of IL-1 $\beta$ and IL-6 in HBE cells induced by miR-152-3p inhibitor. Data shown are mean $\pm S D$ and from three independent experiments. ${ }^{*} P<0.05,{ }^{\star} * P<0.01$. 
A

B

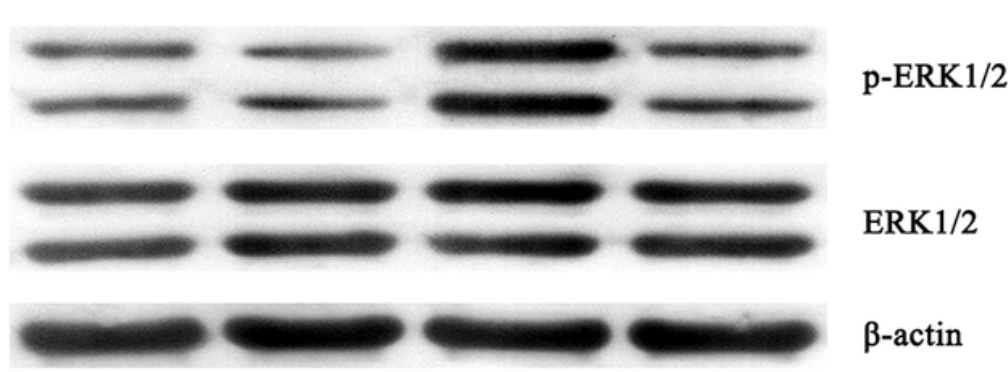

$\begin{array}{lllll}\text { CCAT1-NC } & + & + & - & - \\ \text { CCAT1 } & - & - & + & + \\ \operatorname{mimic}-N C & + & - & + & - \\ \operatorname{miR}-152-3 p & - & + & - & +\end{array}$

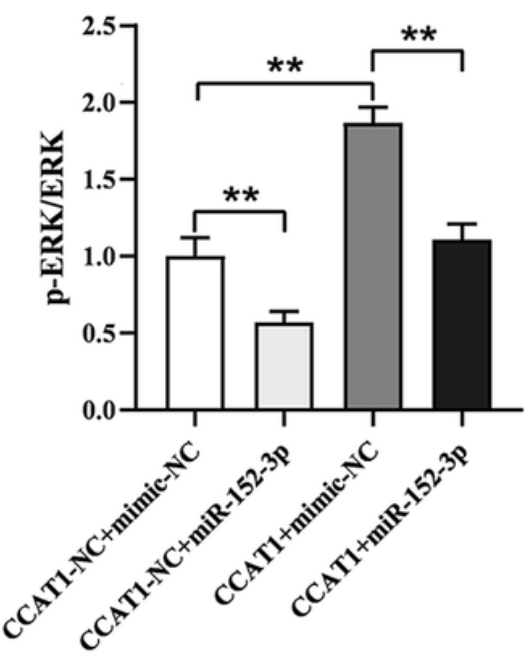

Figure 7

CCAT1 regulates ERK signaling pathway via sponging miR-152-3p. (A,B) Protein expression levels of ERK1/2, p-ERK1/2 in HBE cells co-transfected with CCAT1 vector or CCAT1-NC and miR-152-3p mimic or mimic-NC were analyzed by Western blot. Data shown are mean \pm SD and from three independent experiments. ${ }^{*} \mathrm{P}<0.05,{ }^{*} \mathrm{P}<0.01$. 